\title{
Author Correction: Locoregional delivery of CAR T cells to the cerebrospinal fluid for treatment of metastatic medulloblastoma and ependymoma
}

Laura K. Donovan, Alberto Delaidelli, Sujith K. Joseph, Kevin Bielamowicz, Kristen Fousek (1), Borja L. Holgado, Alex Manno, Dilakshan Srikanthan, Ahmed Z. Gad (1D, Randy Van Ommeren D, David Przelicki, Cory Richman, Vijay Ramaswamy (D), Craig Daniels, Jonelle G. Pallota, Tajana Douglas, Alyssa C. M. Joynt, Joonas Haapasalo, Carolina Nor, Maria C. Vladoiu, Claudia M. Kuzan-Fischer, Livia Garzia, Stephen C. Mack, Srinidhi Varadharajan (D), Matthew L. Baker, Liam Hendrikse (D), Michelle Ly, Kaitlin Kharas, Polina Balin, Xiaochong Wu, Lei Qin, Ning Huang, Ana Guerreiro Stucklin (D, A. Sorana Morrissy (D), Florence M. G. Cavalli, Betty Luu, Raul Suarez, Pasqualino De Antonellis, Antony Michealraj, Avesta Rastan, Meenakshi Hegde, Martin Komosa, Olga Sirbu, Sachin A. Kumar (1), Zied Abdullaev, Claudia C. Faria, Stephen Yip (D), Juliette Hukin (1), Uri Tabori, Cynthia Hawkins, Ken Aldape, Mads Daugaard (1), John M. Maris, Poul H. Sorensen, Nabil Ahmed (1) and Michael D. Taylor (1)

Correction to: Nature Medicine https://doi.org/10.1038/s41591-020-0827-2, published online 27 April 2020.

In the version of this article initially published, a corresponding author (Nabil Ahmed) was not identified as such. The correct citation should include an 'envelope' icon that links to the following email address: nmahmed@texaschildrens.org.

There were 49 duplication or placement errors in BLI images (Figs. 1, 3 and 4 and Supplementary Figs. 2, 3, 4, 6, 7, 10, 13 and 15) and H\&E images (Supplementary Figs. 1, 2, 4, 7 and 15). The correct images are now provided, and a complete list of the images that were corrected is included in the table here. These errors happened during the preparation of the final version of the figures, which comprised over 2,500 BLI and H\&E image panels, and did not affect the results of the paper, as quantification was done using primary data acquired at the same time as the imaging and was independent of images presented in the figures.

\begin{tabular}{|c|c|c|c|}
\hline Figure & Panel (treatment group) & Column & Row \\
\hline Figure 1c & Medulloblastoma: Med114FH $\rightarrow$ TRI CARs & 1 & 2 \\
\hline Figure $3 c$ & Ependymoma: Ep612 $\rightarrow$ HER2 CARs & 4 & 3 \\
\hline Figure $4 c$ & Medulloblastoma: Med114FH $\rightarrow$ i.v. T cell & 3 & 1 \\
\hline Figure $4 c$ & Medulloblastoma: Med114FH $\rightarrow$ i.v. T cell & 4 & 3 \\
\hline Supplementary Figure 1b & Medulloblastoma: Med114FH $\rightarrow$ T cell controls & 1 & 5 \\
\hline Supplementary Figure 1b & Medulloblastoma: Med114FH $\rightarrow$ T cell controls & 3 & 5 \\
\hline Supplementary Figure 2 & Medulloblastoma: Med411FH $\rightarrow$ T cell controls & 6 & 5 \\
\hline Supplementary Figure 2 & Medulloblastoma: Med411FH $\rightarrow$ EPHA2 CARs & 5 & 5 \\
\hline Supplementary Figure 4 & Medulloblastoma: Med114FH Two rounds of TRI-CARs & 7 & 1 \\
\hline Supplementary Figure 4 & Medulloblastoma: Med114FH Two rounds of TRI-CARs & 2 & 1 \\
\hline Supplementary Figure 4 & Medulloblastoma: Med114FH Two rounds of EPHA2 CARs & 3 & 2 \\
\hline Supplementary Figure 4 & Medulloblastoma: Med114FH Two rounds of EPHA2 CARs & 7 & 1 \\
\hline Supplementary Figure 4 & Medulloblastoma: Med114FH Two rounds of EPHA2 CARs & 6 & 1 \\
\hline Supplementary Figure $6 a$ & Ependymoma: MDT-PFA5 $\rightarrow$ TRI CARs & 1 & 1 \\
\hline Supplementary Figure $6 a$ & Ependymoma: MDT-PFA5 $\rightarrow$ TRI CARs & 2 & 1 \\
\hline Supplementary Figure $6 a$ & Ependymoma: MDT-PFA5 $\rightarrow$ TRI CARs & 2 & 2 \\
\hline Supplementary Figure $6 a$ & Ependymoma: MDT-PFA5 $\rightarrow$ TRI CARs & 3 & 1 \\
\hline
\end{tabular}




\begin{tabular}{|c|c|c|c|}
\hline Figure & Panel (treatment group) & Column & Row \\
\hline Supplementary Figure 6a & Ependymoma: MDT-PFA5 $\rightarrow$ TRI CARs & 4 & 2 \\
\hline Supplementary Figure $6 b$ & Ependymoma: MDT-PFA4 $\rightarrow$ HER2 CARs & 1 & 3 \\
\hline Supplementary Figure 6b & Ependymoma: MDT-PFA4 $\rightarrow$ HER2 CARs & 2 & 3 \\
\hline Supplementary Figure 6b & Ependymoma: MDT-PFA4 $\rightarrow$ HER2 CARs & 3 & 3 \\
\hline Supplementary Figure 7a & Medulloblastoma: Med411H IV versus IT single round TRI CARs & 5 & 2 \\
\hline Supplementary Figure 7b & Medulloblastoma: MDT-MMB: IV versus IT single round T cells & 1 & 5 \\
\hline Supplementary Figure 7b & Medulloblastoma: MDT-MMB: IV versus IT single round TRI-CARs & 2 & 1 \\
\hline Supplementary Figure 10a & Medulloblastoma: Med114FH $\rightarrow$ Lateral Ventricle $2.5 \times 10^{6}$ TRI CARs & 1 & 3 \\
\hline Supplementary Figure 10a & Medulloblastoma: Med114FH $\rightarrow$ Lateral Ventricle $2.5 \times 10^{6}$ TRI CARs & 2 & 3 \\
\hline Supplementary Figure 13b & Medulloblastoma: Med411FH T cells + Azacytidine & 4 & 2 \\
\hline Supplementary Figure 13b & Medulloblastoma: Med411FH EPHA2 CARs + Azacytidine & 2 & 4 \\
\hline Supplementary Figure 13b & Medulloblastoma: Med411FH EPHA2 CARs + Azacytidine & 2 & 5 \\
\hline Supplementary Figure 15a & Ependymoma: MDT-PFA4 $\rightarrow$ HER2 CARs + Azacytidine & 7 & 2 \\
\hline Supplementary Figure 15a & Ependymoma: MDT-PFA4 $\rightarrow$ HER2 CARs + Azacytidine & 4 & 3 \\
\hline Supplementary Figure 15a & Ependymoma: MDT-PFA4 $\rightarrow$ TRI CARs + Azacytidine & 6 & 1 \\
\hline Supplementary Figure 15a & Ependymoma: MDT-PFA4 $\rightarrow$ TRI CARs + Azacytidine & 1 & 2 \\
\hline Supplementary Figure 15a & Ependymoma: MDT-PFA4 $\rightarrow$ TRI CARs + Azacytidine & 2 & 2 \\
\hline Supplementary Figure 15a & Ependymoma: MDT-PFA4 $\rightarrow$ TRI CARs + Azacytidine & 3 & 2 \\
\hline Supplementary Figure 15a & Ependymoma: MDT-PFA4 $\rightarrow$ TRI CARs + Azacytidine & 3 & 4 \\
\hline Supplementary Figure 15b & Ependymoma: Ep612 $\rightarrow$ TRI CARs + Azacytidine & 6 & 2 \\
\hline Supplementary Figure 15b & Ependymoma: Ep612 $\rightarrow$ HER2 CARs + Azacytidine & 2 & 4 \\
\hline Supplementary Figure 15b & Ependymoma: Ep612 $\rightarrow$ T cells + Azacytidine & 3 & 4 \\
\hline
\end{tabular}

The legends to Figs. 2b, 4b, 5b and $6 \mathrm{~b}$ did not acknowledge that data from experimental groups first shown in Fig. 1 had been replotted for comparison purposes. The correct panel legends end as follows, to include information about which experimental groups were replotted: "Data from experimental groups 'no treatment, 'single treatment non-transduced T-cells', 'single treatment TRI CAR T-cells' and 'single treatment EPHA2 CAR T-cells', first plotted in Fig. 1, were replotted in Fig. 2 as experimental comparisons" (Fig. 2b); "Data from experimental groups 'no treatment, 'single treatment non-transduced T-cells', 'single treatment TRI CAR T-cells' and 'single treatment EPHA2 CAR T-cells', first plotted in Fig. 1, were replotted in Fig. 4 as experimental comparisons" (Fig. 4b); "Data from experimental groups 'no treatment, 'single treatment non-transduced T-cells', 'single treatment TRI CAR T-cells' and 'single treatment EPHA2 CAR T-cells', first plotted in Fig. 1, were replotted in Fig. 5 as experimental comparisons" (Fig. 5b); and "Data from experimental groups 'no treatment, 'single treatment non-transduced T-cells', 'single treatment TRI CAR T-cells' and 'single treatment EPHA2 CAR T-cells', first plotted in Fig. 1, were replotted in Fig. 6 as experimental comparisons” (Fig. 6b).

In Extended Data Fig. 2d,f, the H score in the bottom right corner of each image was incorrect. The original and corrected images are provided here.

In Extended Data Fig. 4g, the label for the bottom row ('Normal Paediatric Thalamus Tissue') was incorrect; the correct label is 'Normal Paediatric Thalamus Opticus'.

The following subsection of the Methods section was missing, immediately after the subsection 'Orthotopic injection of tumor cells and bioluminescent imaging':

\section{Multiplex cytokine analysis}

Soluble serum and CSF cytokines from animals treated with $2.5 \times 10^{6}, 5.0 \times 10^{6}$ and $10 \times 10^{6}$ EPHA2 CAR T-cells, TRI CAR T cells or non-transduced $\mathrm{T}$ cells, delivered intraventricularly via the lateral ventricle, or intravenously via the tail vein, were quantified using the human magnetic Luminex 18-Plex Assay (LXSAHM - MCP-1, MIP-1 $\alpha$, GM-CSF, IFN- $\gamma$, IL-1 $\beta$, IL-2, IL-4, IL-5, IL-6, IL-7, IL-8, IL-10, IL-12p70, IL-13, IL-15, IL-17A, IL-23, TNF $\alpha$ ) and the mouse magnetic Luminex 6-Plex Assay (LXSAMSM - GM-CSF, IFN- $\gamma$, IL-1 $\beta$, IL-6, LIX, TNF $\alpha$ ) according to the manufacturer's instructions (R\&D Systems). Briefly, $25 \mu \mathrm{l}$ and $10 \mu \mathrm{l}$ (made to $50 \mu \mathrm{l}$ with diluent) of serum and CSF, respectively, were incubated in a 96-well plate with capture magnetic beads overnight at $4{ }^{\circ} \mathrm{C}$ on a microplate shaker. The following day, $50 \mu \mathrm{l}$ of diluted biotin-antibody cocktail was added to the wells and incubated at room temperature for an hour. The assay was developed by adding $50 \mu \mathrm{l}$ of diluted Streptavidin-PE and data acquired on a Luminex-200 instrument with xPONENT 4.3 software. Analysis was performed using R-studio and two-sided one-way analysis of variance (ANOVA) followed by Tukey post hoc test.

All errors have been corrected in the HTML and PDF versions of the article. 


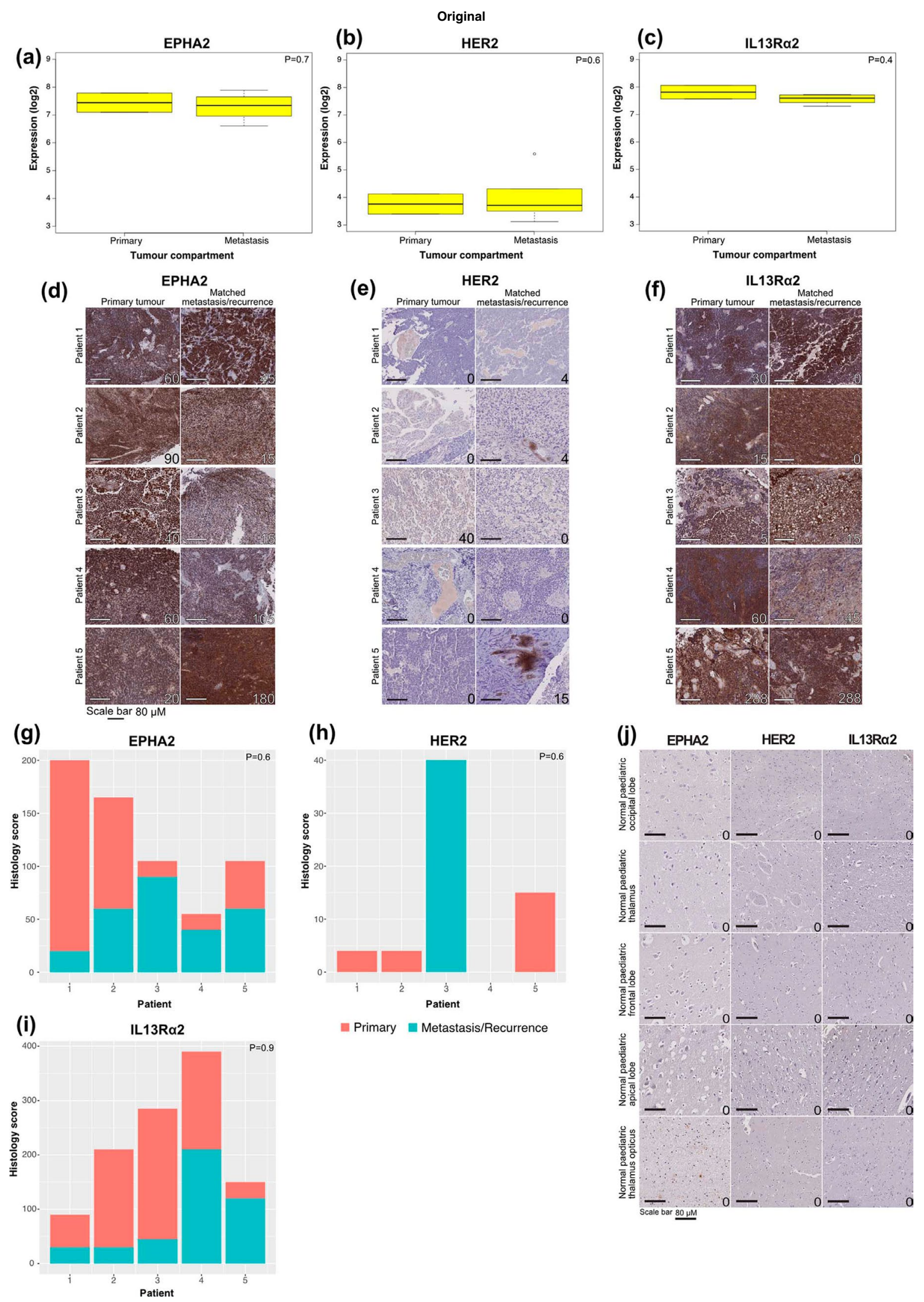

Extended Data Fig. 2 | Original and corrected. 

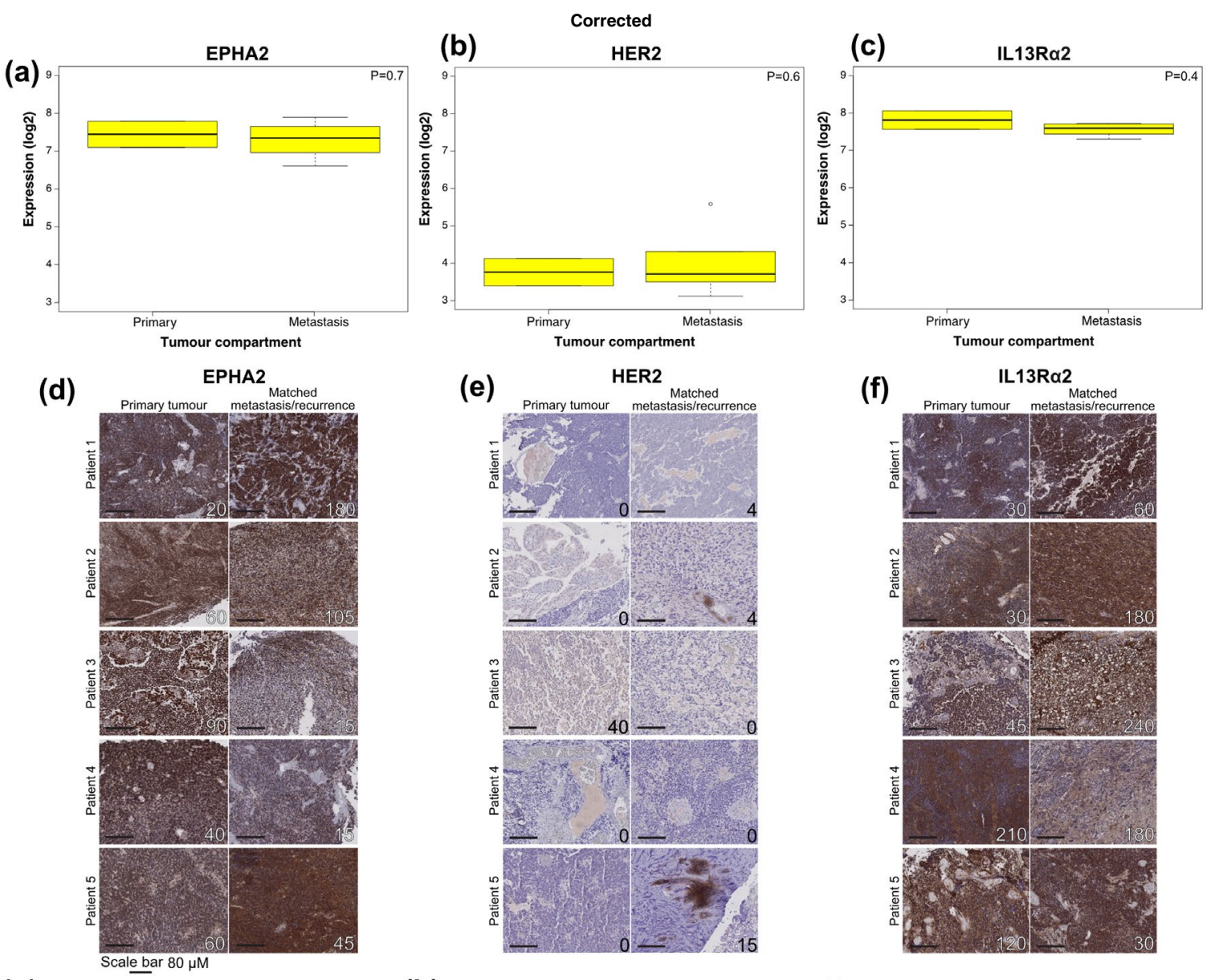

(e)

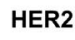

(f)

IL13Ra2
Matched
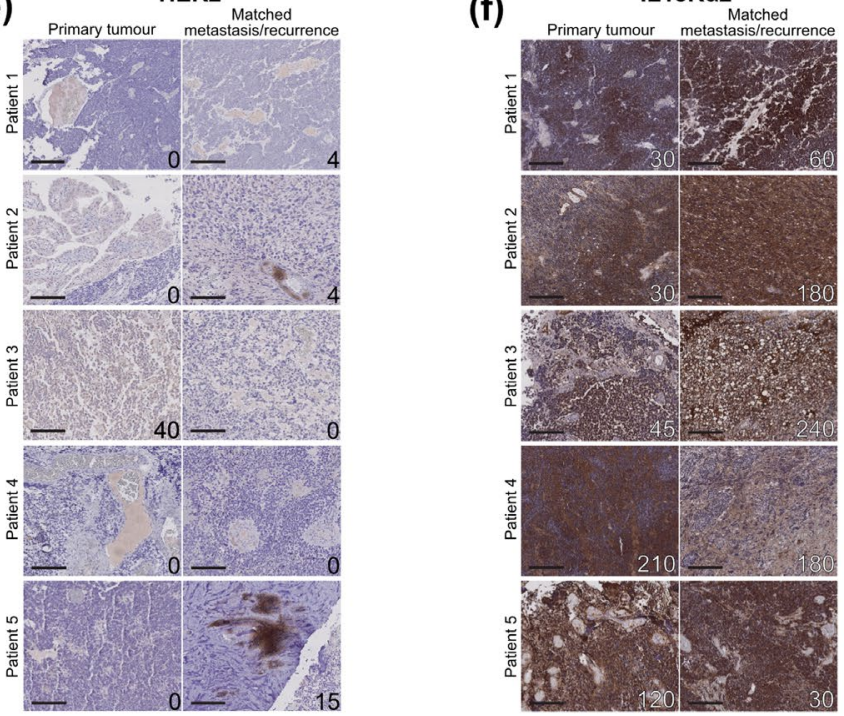

(g)

EPHA2

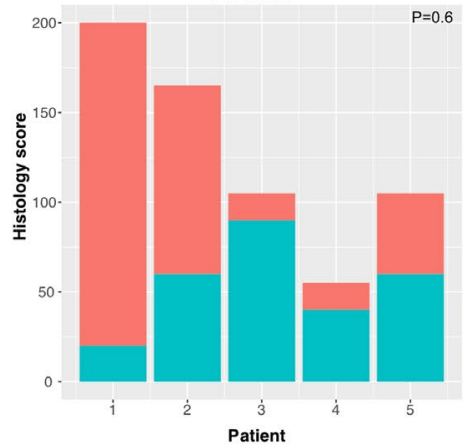

(h)

HER2
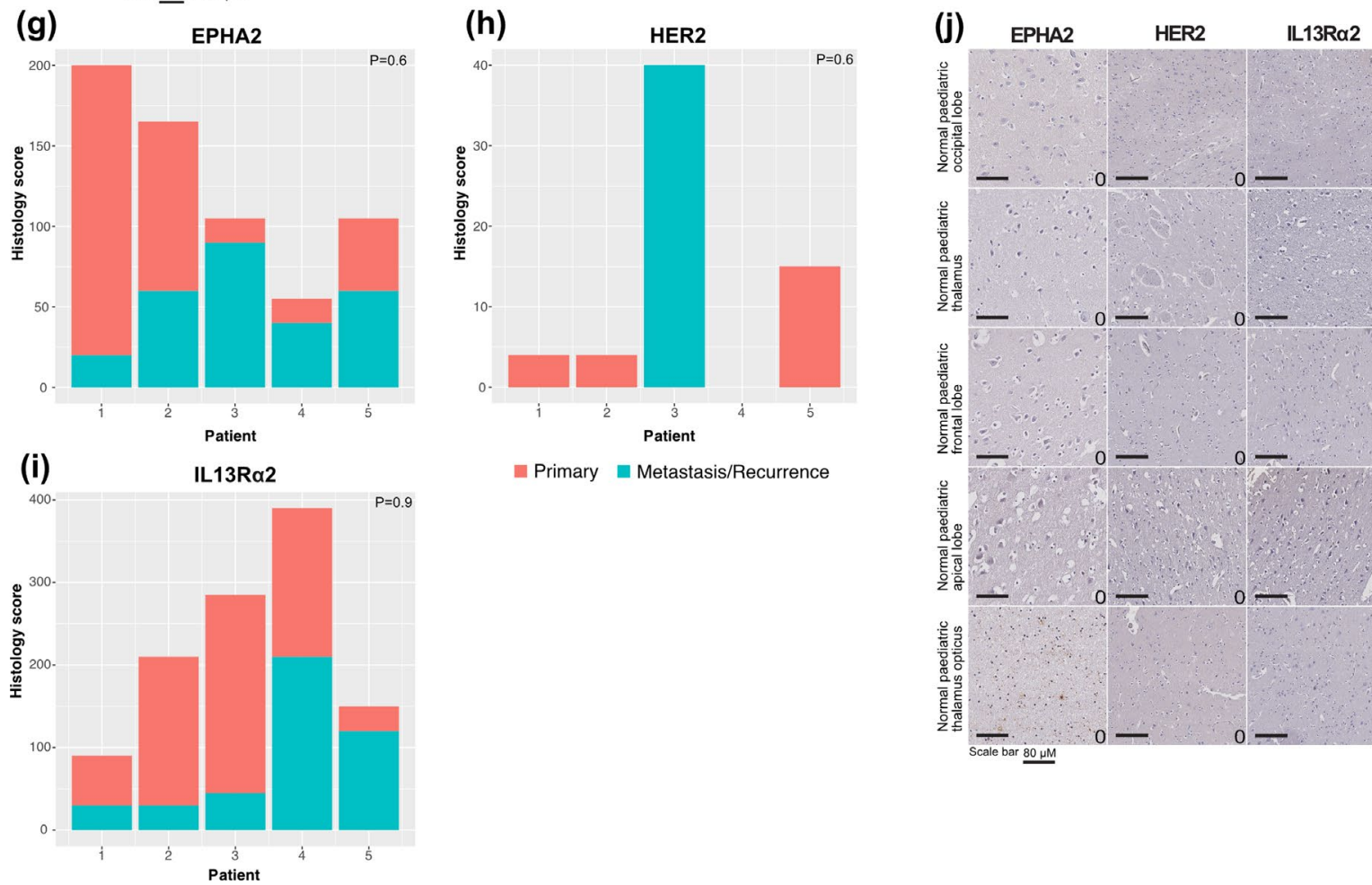

arimary Metastasis/Recurrence

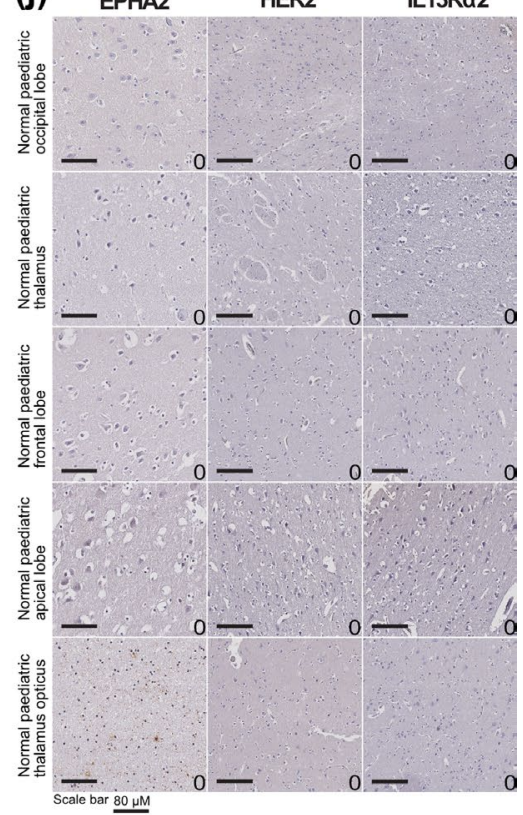

Published online: 27 May 2021

https://doi.org/10.1038/s41591-021-01362-1

(c) The Author(s), under exclusive licence to Springer Nature America, Inc. 2021 